\title{
Retinal Compression Folds
}

\author{
J. M. TWOMEY and P. K. LEAVER \\ London
}

\begin{abstract}
Summary
Twelve cases of retinal compression folds occurring after successful retinal re-attachment surgery are reported. In all 12 eyes surgery included the use of a circumferential buckle, internal tamponade with gas or silicone oil and drainage of sub-retinal fluid. The mechanism of retinal fold formation is discussed.
\end{abstract}

Internal tamponade of retinal breaks is commonly used in the surgery of acute retinal detachments, particularly in the presence of large or posterior tears with bullous detachment. ${ }^{1}$ Air or air-gas mixtures are most often used for this purpose although silicone oil is indicated for giant retinal tears. ${ }^{2}$ Vitrectomy is frequently though not always undertaken to improve access ${ }^{3}$ and to facilitate fluid/gas or fluid/silicone oil exchange. We report the occurrence post-operatively of flat retinal folds in some cases of successful re-attachment of acute retinal detachments using internal tamponade, similar to that described by Pavan in a single case. ${ }^{4}$

\section{Findings}

A series of 12 eyes in 12 patients in whom large flat falciform retinal folds were noted after successful surgery for acute bullous retinal detachments is reported.

In all 12 eyes a circumferential buckle was used to support the breaks; an encircling band alone in three, a band and a tyre in five and a tyre alone in four. Three eyes underwent gas injection after drainage of sub-retinal fluid alone, in seven fluid/gas exchange was carried out after a vitrectomy and in two vitrectomy and fluid/silicone oil exchange was undertaken. Sub-retinal fluid was drained externally in four eyes, internally in

TABLE I Surgical methods used in 12 cases

\begin{tabular}{lllll}
\hline CASE & BUCKLE & VITRECTOMY & DRAIN & TAMPONADE \\
\hline 1 & Band and Tyre & Yes & Int & Gas \\
2 & Band & Yes & Int & Gas \\
3 & Band and Tyre & Yes & Int & Gas \\
4 & Band & Yes & Int & Gas \\
5 & Band and Tyre & Yes & Ext & Gas \\
6 & Band & Yes & Int & Gas \\
7 & Band and Tyre & Yes & Both & Oil \\
8 & Tyre & Yes & Int & Oil \\
9 & Band and Tyre & Yes & Int & Gas \\
10 & Tyre & No & Ext & Gas \\
11 & Tyre & No & Ext & Gas \\
12 & Tyre & No & Ext & Gas \\
\hline
\end{tabular}



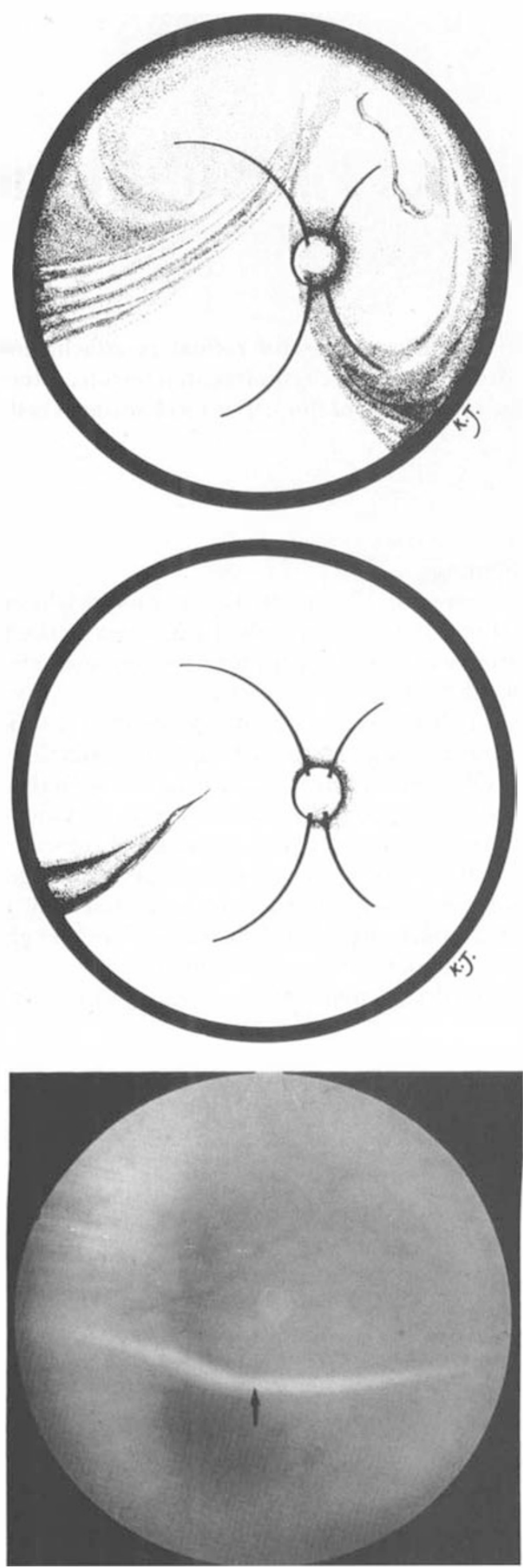

seven and via both routes in one. Cryotherapy through full thickness sclera was applied to the retinal breaks in all cases. (Table I)

The folds ran radially, either anteriorly from the disc or posteriorly from the buckle and the macula was involved in three eyes, limiting visual recovery. These features are illustrated in the following two cases.

Case 2: Mrs AC, a 61 year old lady, presented in July 1986 with a dense vitreous haemorrhage and superior retinal detachment following blunt trauma to her right eye. Visual acuity was 6/24 right and 6/6 left and a large, ragged retinal tear was found at surgery in the supero-nasal quadrant. Vitrectomy with encircling band, cryotherapy and internal fluid-gas exchange was undertaken, a bulla of residual sub-retinal fluid being noted at the end of the procedure. She was postured on her right side during the post-operative period and the following day was noted to have a retinal fold at what had been the infero-temporal extremity of the detached retina. A large gas bubble, filling most of the pre-retinal space, was measured postoperatively using an ultra-sound technique. ${ }^{5}$ (Fig. 1)

Case 3: Mr. RA, a 45 year old man with a history of injury to his left eye when aged 12 years, presented in January 1983 with visual loss in his left eye for one week. He was found to have a subtotal retinal detachment with a large temporal retinal break. Visual acuity was $6 / 5$ right and perception of light left. An internal fluid-gas exchange was carried out after vitreo-lensectomy and the break was supported with an encircling band and tyre. He was postured on his right side post-operatively and the following day 2 folds radiating anteriorly from the disc were noted in an otherwise flat retina. One fold ran to the buckle on the nasal side while the temporal fold crossed the macula. His final vision was $6 / 60$ left with the aid of a contact lens. (Fig. 2)

\section{Discussion}

The formation of post-operative retinal folds implies that there has been either an increase in retinal surface area consequent upon the detachment itself or that the operation has

Fig. 1. Case 2. a. pre-operative retinal drawing. b. post-operative retinal drawing ilustrating position of retinal fold.

c. fundus photograph showing retinal fold. 
created redundant retina, or both. Goldman in $1975^{6}$ explaining the mathematics of scleral buckling and Pruett in $1977^{7}$ discussing the fishmouth phenomenon describe how retinal redundancy occurs.

A scleral buckle has the effect of elongating the wall of the eye perpendicular to it's

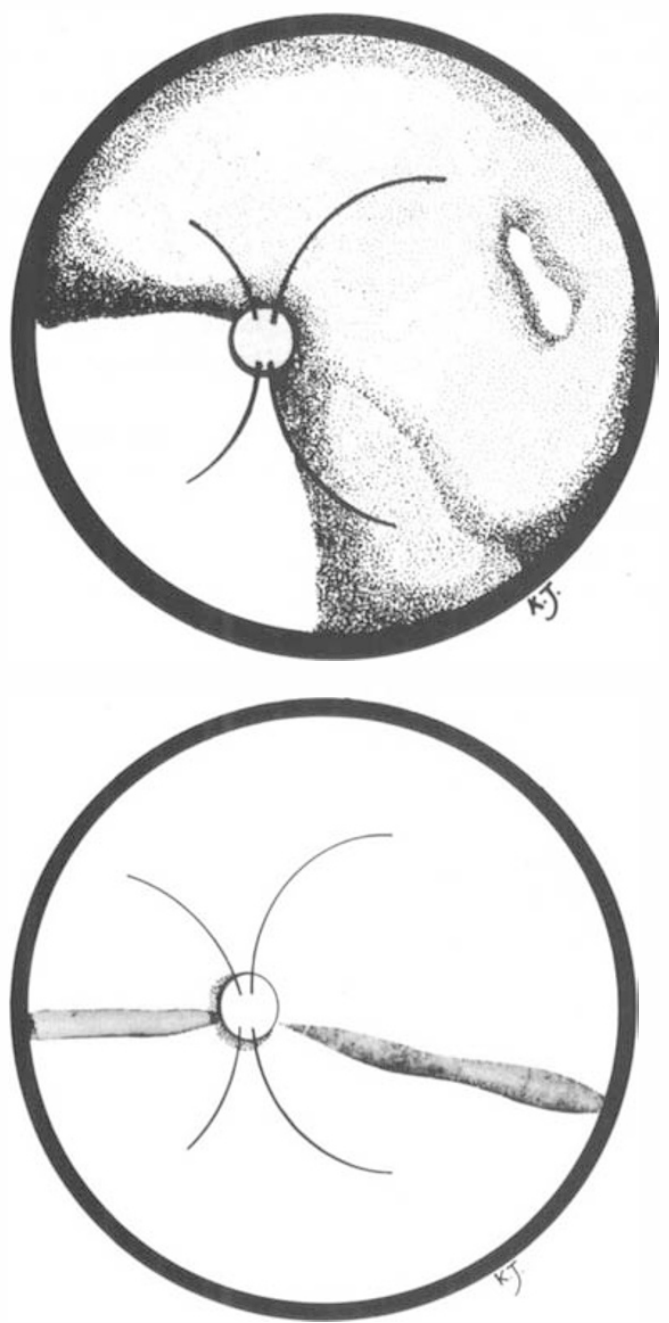

Fig. 2. Case 3. a. pre operative retinal drawing. b. post-operative retinal drawing illustrating position of retinal folds.

c. fundus photograph showing retinal folds extending nasally and temporally from the optic disc.

$d$. fundus photograph of nasal retinal fold extending posteriorly from the buckle.

e. fundus photograph showing temporal retinal fold crossing the macula.
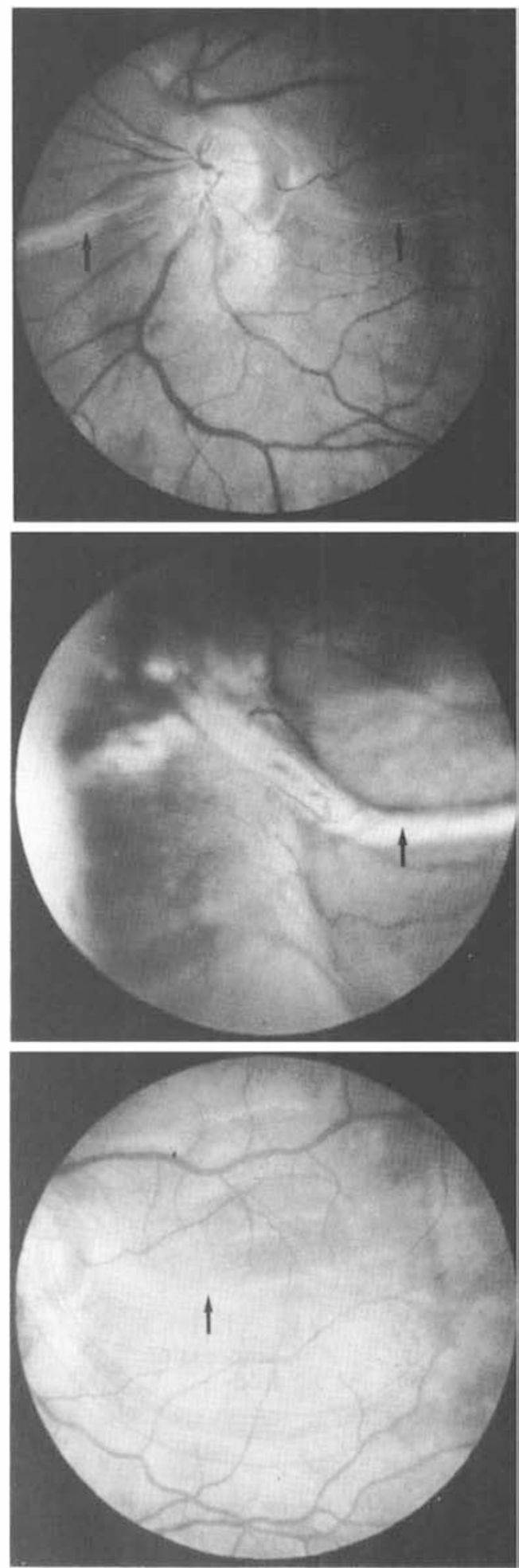
long axis such that on re-attachment the retina is relatively shortened and under tension in this plane. At the same time, the reduction in the radius of curvature of the eye (r) under the buckle can lead to a reduction in the circumference of the inner eye wall (c) relative to the detached retina. $(c=2 \pi r)$. This change occurs along the long axis of the explant and can create redundant retina in this meridian on retinal re-attach-
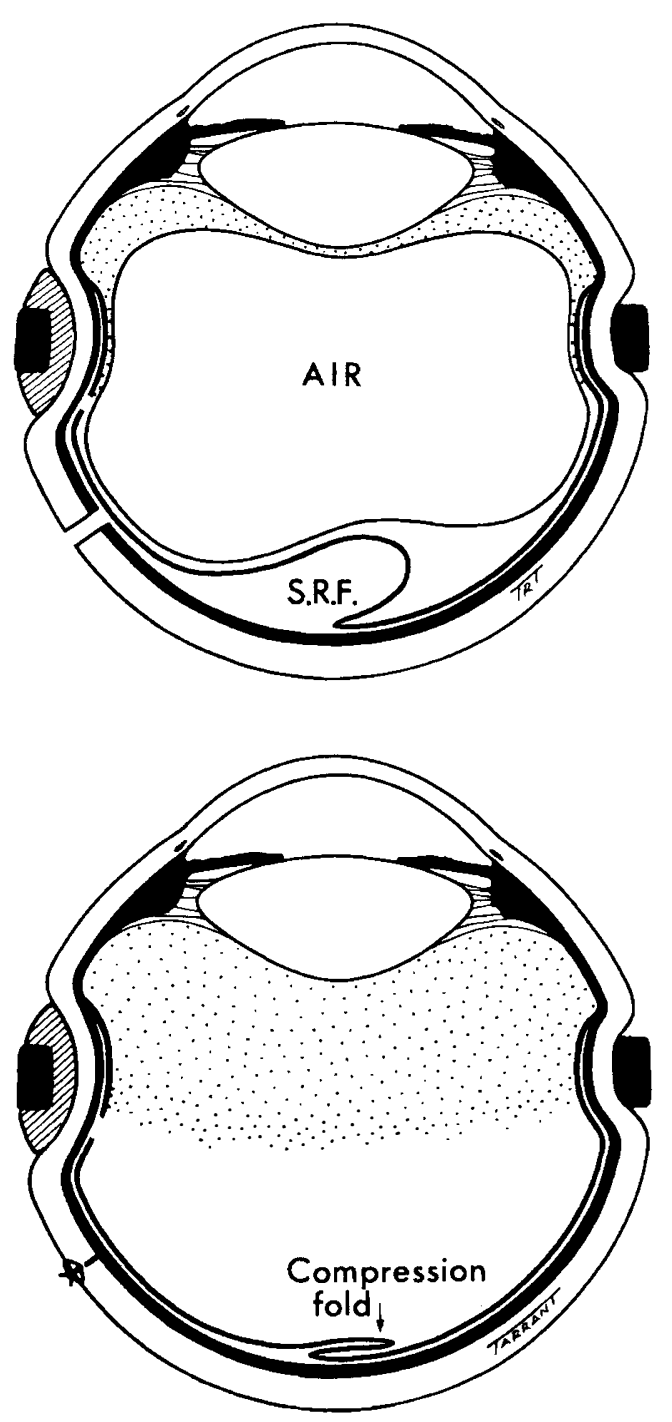

Fig. 3. $a$ and $b$. Drawings to demonstrate sequestration of sub-retinal fluid from the drain site by a bubble of gas (a) and formation of a fold. (b). ment. These effects are more pronounced with circumferential than with radial buckles, the retina being relatively shortened in an antero-posterior direction with an associated circumferential retinal redundancy.

Retinal redundancy is proportional to the length, breadth and height of the buckle and the surplus retina so created is usually accommodated by remodelling, although retinal folds may sometimes radiate from the edge of the buckle and cause fishmouthing of retinal tears?

The folds we describe, like that reported by Pavan, ${ }^{4}$ are usually single, flat and often large and we propose that the behaviour of the redundant retina caused by the buckle has been modified by the presence of gas or oil injected for the purpose of internal tamponade.

They occur at the posterior and inferior extremities of detached retinal bullae because large bubbles of gas or globules of oil isolate residual sub-retinal fluid from the drain site and compress the associated redun-

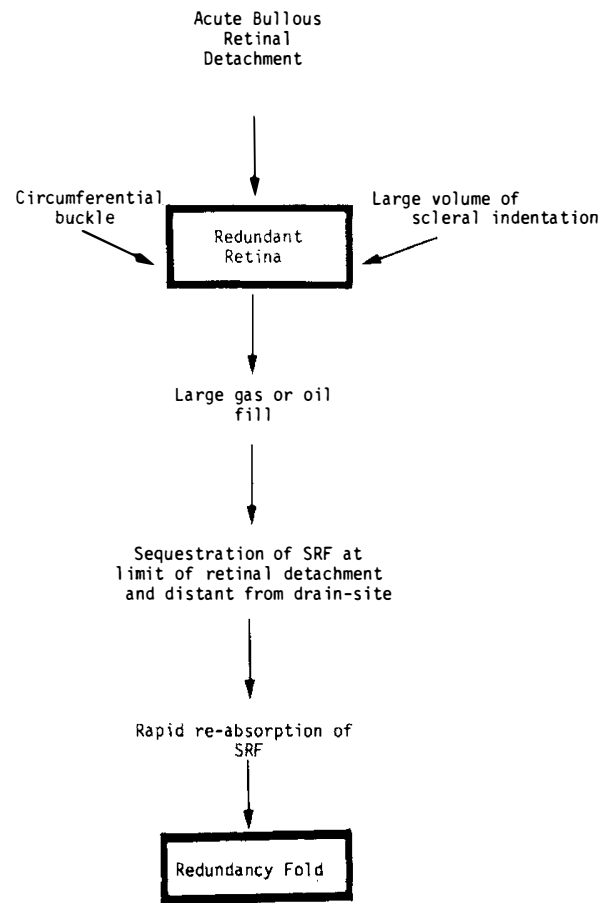

Fig. 4. Proposed sequence of events leading to fold formation. 
dant retina at the limits of the detachment. (Fig. 3) Sequestration of sub-retinal fluid from the drain site, whether internal or external, is more likely to occur when large bubbles of gas or globules of oil are injected into the pre-retinal space and the presence of residual fluid predisposes to fold formation. When rapid re-absorption of sub-retinal fluid occurs after effective closure of the break, the displaced and redundant retina which would otherwise re-model or form several small folds from the posterior border of the buckle may be compressed by the bubble or globule and fold on itself in the immediate post-operative period. (Fig. 4)

In long standing retinal detachments where post-operative fluid re-absorption is slow, ${ }^{8}$ the redundant retina has time to re-model as the fluid re-absorbs, so that these folds rarely occur. Likewise, when smaller volumes of gas are injected during non-drainage procedures, pockets of sub-retinal fluid are not usually isolated from the retinal break, nor have we seen these folds in the absence of a buckle, which suggests that retinal elasticity alone is insufficient to account for them.

Visual outcome in this series of eyes complicated by such redundancy folds was seriously affected in only 3 out of 12 , in which the fold crossed the macula. Nevertheless, care should be taken when using large volumes of agents for internal tamponade in cases of acute bullous retinal detachment, to limit the volume of scleral indentation, drain adequately sub-retinal fluid and to posture the patient appropriately in the post-operative period.

We are grateful to Miss Karen Johnstone of the Department of Medical Illustration, Institute of Ophthalmology, and Mr. T. Tarrant for their help with illustrations and to Mr. D. Mcleod and Mr. Z. Gregor for allowing us to study their cases.

\section{References}

${ }^{1}$ Norton EWD: Intra-ocular gas in the management of selected retinal detachments. Trans. Am. Acad. Ophthalmol. Otolaryngol. 1973; 77: OP 85-98.

2 Lean JS, Leaver PK, Cooling RJ, McLeod D: Management of complex retinal detachments by Vitrectomy and fluid-silicone exchange. Trans. Opthalmol. Soc. UK. 1982; 102: 203-5.

${ }^{3}$ Ratner CM, Michels RG, Auer C, Rice TA. Pars plana Vitrectomy for complicated retinal detachments. Opthalmology. 1983; 90: 13237.

${ }^{4}$ Pavan P. Retinal fold in macula following intraocular gas; an avoidable complication of retinal detachment surgery. Arch. Opthalmol. 1984; 102: 83-4.

${ }^{5}$ Jacobs PM. Intra-ocular gas measurement using A-scan ultra-sound. Curr. Eye Res. 1986; 5: 575-8.

${ }^{6}$ Goldbaum MH, Smithline M, Poole TA, Lincoff HA. Geometric analysis of radial buckling. Am. J. Ophthalmol. 1975; 79: 958-65.

${ }^{7}$ Pruett RC. The Fishmouth phenomenan 2. Arch Ophthalmol. 1977; 95: 1782-7.

${ }^{8}$ Leaver PK, Chester GH, Saunders SH. Factors influencing absorption of sub-retinal fluid. $\mathrm{Br}$. J. Ophthalmol. 1976; 60: 557-60. 